ISSN: 2773-7349

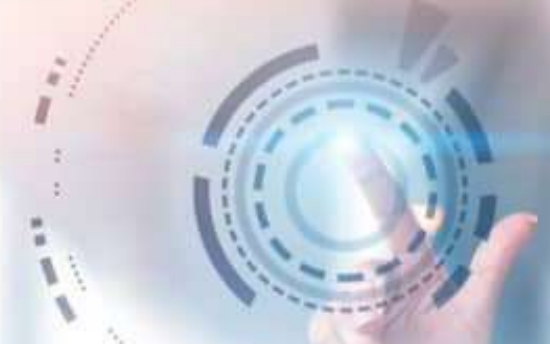

Sociedad \& Tecnología

Revista del Instituto Tecnológico Superior Jubones

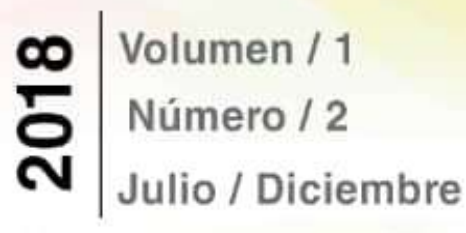

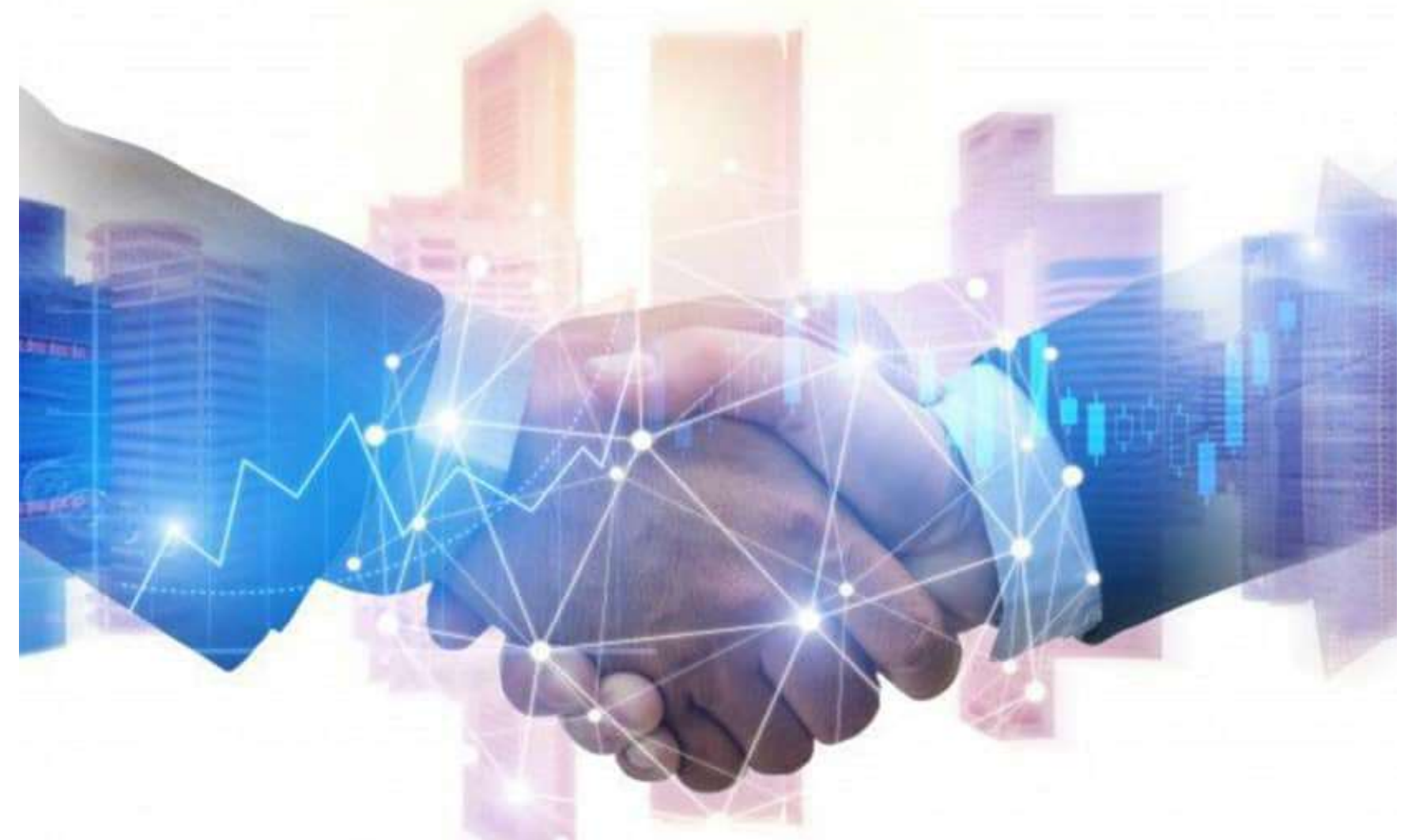




\title{
EL TRABAJO EDUCATIVO PARA PREVENIR LA VIOLENCIA EN ADOLESCENTES CON DISCAPACIDAD INTELECTUAL MODERADA
}

\author{
EDUCATIONAL WORK TO PREVENT VIOLENCE IN ADOLESCENTS WITH MODERATE INTELLECTUAL DIS
}

Teresa Nápoles Gómez

E-mail: tnapoles@uo.edu.cu

ORCID: https://orcid.org/0000-0001-5434-8641

E-mail: Daylanis Gutiérrez Cruzata

E-mail: dgutierrez@uo.edu.cu

ORCID: https://orcid.org/_0000-0003-4855-4814

Universidad de Oriente, Cuba

Cita sugerida (APA, séptima edición)

Nápoles Gómez, T., \& Gutiérrez Cruzata, D. (2018). El trabajo educativo para prevenir la violencia en adolescentes con discapacidad intelectual moderada. Revista Sociedad \& Tecnología, 1(2), 1118.

\section{RESUMEN}

En la Agenda 2030 para el desarrollo sostenible se establece en el objetivo 5 las metas para prevenir y eliminar todas las formas de violencia contra las mujeres y las niñas; en tal sentido la presente investigación va dirigida a revelar esta problemática en las atletas con discapacidad intelectual moderada del equipo de Gimnasia Rítmica de Santiago de Cuba que tienen trece y catorce años, teniendo como objetivo la aplicación de acciones educativasterapéuticas para la prevención de actos de violencia en las atletas. A través del trabajo educativo realizado en el proceso de entrenamiento se logró identificar, caracterizar y transformar las conductas de las mismas, desde el sistema de influencias educativas para prevenir la violencia en las estudiantes- atletas. El estudio realizado por las autoras del presente trabajo tuvo un periodo de observación y aplicación de actividades de 8 meses durante el entrenamiento del equipo, que unido a otros métodos científicos tales como la entrevista y la observación permitieron corroborar el problema y aplicar el objetivo. Los resultados obtenidos muestran cambios en el comportamiento de las atletas desde el punto de vista físico, estético, biológico y psicológico que se consideran las conclusiones parciales de esta experiencia pedagógica.

\section{Palabras clave:}

prevención, violencia, discapacidad intelectual moderada, trabajo educativo, género.

\begin{abstract}
The 2030 Agenda for Sustainable Development establishes goals to prevent and eliminate all forms of violence against women and girls in Goal 5; In this sense, this research is aimed at revealing this problem in athletes with moderate intellectual disabilities of the Rhythmic Gymnastics team of Santiago de Cuba who are thirteen and fourteen years old, aiming at the application of educational-therapeutic actions for the prevention of acts of violence in athletes. Through the educational work carried out in the training process, it was possible to identify, characterize and transform their behaviors, from the system of educational influences to prevent violence in studentathletes. The study carried out by the authors of the present work had a period of observation and application of activities of 8 months during the training of the team, which together with other scientific methods such as interview and observation allowed to corroborate the problem and apply the objective. The results obtained show changes in the behavior of the athletes from the physical, aesthetic, biological and psychological point of view that are considered the partial conclusions of this pedagogical experience.
\end{abstract}




\section{Keywords:}

prevention, violence, moderate intellectual disability, educational work, gender.

\section{INTRODUCCIÓN}

Desde el triunfo revolucionario en Cuba se comenzó a trabajar con el objetivo de romper con los clásicos y rígidos estereotipos que limitan y despersonalizan, para lograr el ejercicio de la equidad entre los géneros, proponiendo alcanzar formas de expresión, relación más plenas y responsables. Se promovió la incorporación de la mujer a cada una de las actividades del quehacer social. El reconocimiento a sus derechos propios, el enjuiciamiento a la posición social que ocupaba, la revalorización de sus potencialidades y sus capacidades, la garantía de la igualdad de oportunidades y las condiciones que aseguran su realización personal y social, por lo que respecto al mundo llevamos una gran ventaja (Barrios, 2002).

Nuestro comandante en jefe calificó el protagonismo femenino como"... una Revolución dentro de otra Revolución" por el alcance de las transformaciones en la vida y en el pensamiento de las mujeres cubana, que se hizo fuerte a través de la Federación de Mujeres Cubanas movimiento que impulsa su promoción y participación en todas las esferas y niveles de esta sociedad (Fuentes, 2018).

Desde los círculos infantiles y las escuelas se trazan políticas para la equidad e igualdad entre los niños y las niñas siendo protegidos de actos de violencias, maltrato, burlas y el estado ha prestado particular atención al tema de la discapacidad por lo que ha implementado una estrategia de desarrollo fundada en los principios de libertad, equidad, justicia social e inclusión en medio de dificultades derivadas del incesante e inhumano bloqueo económico, comercial y financiero, impuesto a Cuba por el Gobierno de los Estados Unidos durante más de 50 años, de las limitaciones económicas y financieras resultantes de su condición de país en desarrollo y del impacto devastador de los fenómenos climatológicos (Cobas, 2010).

La Agenda 2030 para el Desarrollo Sostenible, aprobada en septiembre de 2015 por la Asamblea General de las Naciones Unidas, establece en su objetivo 5, lograr la igualdad entre los géneros y empoderar a todas las mujeres y las niñas trazando como meta "eliminar todas las formas de violencia contra todas las mujeres y las niñas en los ámbitos público y privado, incluidas la trata y la explotación sexual y otros tipos de explotación".

Nuestro país promueve la necesidad de la inclusión trazando vías, herramientas, proyectos para que las personas con discapacidad intelectual logren ser autogestores de su desarrollo personal y sus procesos de inclusión con la reafirmación del derecho fundamental de todos los niños/as y jóvenes a acceder a la educación, al deporte, la recreación reconociendo que cada uno de ellos posee características, intereses, capacidades, potencialidades y necesidades de aprendizajes que le son propios (GAFDI, 2017).

Es reconocido el papel del deporte como factor de rehabilitación mental, física, emocional y social. En Cuba la práctica sistemática del mismo siempre ha sido considerada como un elemento esencial para el logro de la salud lo cual es un logro mancomunado del INDER, MINSAP y MINED en la proyección de estrategias y planes para la incorporación a los diferentes deportes adaptados. No hay dudas de la importancia que tiene la incorporación al deporte para los discapacitados cuyo propósito fundamental es el mejoramiento de la calidad de vida y la inclusión social (Colectivo de autores, 2017).

Las escuelas especiales tienen como objetivo primordial, prepararlos para la vida adulta e independiente. Entre sus intenciones se encuentra en alentar al movimiento de Olimpiadas especiales cuya misión es proporcionar entrenamiento deportivo y competición atlética durante todo el año en una variedad de deportes olímpicos, desde los 8 años de edad dándoles la oportunidad de desarrollar la aptitud física, demostrar coraje, experimentar alegría y participar en un intercambio de premios, destrezas y compañerismo con sus familias, otros atletas especiales y la comunidad (Chamero, 2016).

Distintos estudios han llegado a la conclusión de que las personas con discapacidad intelectual moderada son discriminadas en la participación de actividades físicas y del deporte ya que han demostrado barreras específicas tales como inaccesibilidad a los complejos deportivos y equipamientos, a la participación en actividades deportivas, ausencia de conocimientos sobre entrenamiento, actitudes y conductas negativas impidiendo su desarrollo pleno.

Estos demuestran además que las adolescentes con discapacidad intelectual sufren

Volumen 1 | número 2 | julio-diciembre | 2018 
discriminación por su discapacidad y a pesar de ser niñas ya la padecen, no sólo tienen que luchar contra las barreras y retos tradicionales que se encuentran las adolescentes en la familia, en la comunidad, en el deporte y en el mundo escolar sino que además, padecen las dificultades adicionales, obstáculos y falta de oportunidades, derivadas de la propia discapacidad, discriminándola en las necesidades y aspiraciones propias de los adolescentes junto con un mayor riesgo de padecer abuso sexual, psicológico $\mathrm{y} / \mathrm{o}$ físico (Miranda, 2018).

Entre los deportes practicados se incluye la Gimnasia Rítmica donde el equipo de Santiago de Cuba de la categoría 13-14 años son niñas que pertenecen a la escuela especial Manuel Isla, las mismas presentan discapacidad intelectual moderada que de acuerdo a esta patología, es entendida como una condición en la que las personas que la padecen tienen severas limitaciones y deficiencias en el funcionamiento intelectual teniendo problemas en el razonamiento, la planificación, la resolución de problemas o el aprendizaje y en el desarrollo cognoscitivo en general cuyas limitaciones funcionales son compensadas por los apoyos disponibles de su entorno (Cubamotricidad, 2018).

En tal sentido este trabajo va dirigido al trabajo educativo en el proceso de entrenamiento cuyo objetivo se centra en aplicar acciones educativas terapéuticas para prevenir actos de violencia en las atletas de Gimnasia Rítmica de Santiago de Cuba (Libardoni, 2006). Esta investigación va dirigida a contribuir, identificar, caracterizar y transformar las conductas de las mismas, desde el sistema de influencias educativas para prevenir la violencia en las estudiantes- atletas. Además de percibir e identificar actos de violencia para la búsqueda de ayuda ya que esta población es la más vulnerable a sufrir abuso sexual, maltrato y negligencia familiar y escolar.

\section{MATERIALES Y MÉTODOS}

Los resultados de las técnicas e instrumentos aplicados, permitieron trazar las estrategias de trabajo educativo, encaminada a enfrentar las insuficiencias y brindar alternativas que aseguren la más completa y eficiente atención a estas adolescentes en el enfrentamiento $y$ prevención de actos de violencia.

Se realizó con una población de 4 atletas del equipo de Gimnasia Rítmica de Santiago de
Cuba representando el $100 \%$ de la muestra de las atletas de esta categoría juvenil.

La muestra de esta investigación presenta discapacidad intelectual moderada, perteneciente al sexo femenino con una edad entre 13-14, donde cursan los grados de sexto y séptimo. Estas estudian en la escuela especial Manuel Enrique Isla Pérez que acoge niños y niñas con esta necesidad educativa que se encuentra enclavada en el Consejo Popular Manuel Isla, Micro 7, Distrito José Martí.

Estas adolescentes se caracterizan por ser cariñosas, alegres, les gustan las actividades físicas, al igual que el deporte que practican. Son entusiastas, sin embargo no se motivan con facilidad y son hiperactivas. En cuanto a las características intelectuales, presentan dificultades para aprender, adquirir conocimientos, lograr su dominio y representación. Presentan dificultades en el lenguaje correcto y a veces incoherente, de pensamiento concreto teniendo problemas en el razonamiento y su comunicación. Además presentan deformidades posturales evaluada en una cifosis.

Se tomaron en cuenta los métodos teóricos como análisis-síntesis, inductivo-deductivo y sistémico estructural y los métodos empírico revisión documental, la observación y la entrevista como técnica.

En la observación realizada y las entrevistas a las atletas y familiares se corrobora que han sufrido actos de violencia física y psicológica en cuanto a burlas al presentar las coreografías, hombres adultos que cuando saben que practican este deporte ven la flexibilidad que presentan y la instan a estar con ellos con promesas materiales.

Las autoras de esta investigación asumen que estas acciones educativa-terapéuticas al combinarlas en el proceso de entrenamiento de la Gimnasia Rítmica que se expresa mediante movimientos a partir de órdenes cortas y precisas; acompañadas de música desarrollan sentimientos, forman costumbres y hábitos de conducta; lo cual se logra, de manera general, siempre que exista una influencia sistemática y planificada en el proceso.

La Gimnasia Rítmica en las Olimpiadas especiales es una disciplina especialmente desafiante ya que demanda destreza y expone a riesgos por la manipulación simultánea de objetos, este deporte implica el trabajo del sistema muscular y esquelético en su totalidad. 
Su objetivo fundamental es la integración de las personas con discapacidad en contextos normalizados, además de aportar los beneficios que conlleva la Gimnasia Rítmica como el aumento de autonomía, independencia y desarrollo personal.

Estas acciones tienen como objetivo orientar a las adolescentes hacia un comportamiento adecuado, a partir del acatamiento de las normas, los valores sociales y detectar indicios de violencia para solicitar la ayuda necesaria.

Se aplicaron en las etapas de preparación especial y pre competitivo con un tiempo de duración de 8 meses y una frecuencia de cinco días a la semana.

Teniendo en cuenta que su aprendizaje es lento y limitado en las sesiones de entrenamiento en coordinación con la entrenadora se ofrecían elementos que brinda el trabajo educativo para que ellas puedan identificar, reconocer signos de acoso sexual, de maltrato físico y psicológico mediante dramatizaciones, láminas, medios audiovisuales.

Partiendo de que el trabajo educativo se concreta en todas aquellas acciones e influencias conscientes, dirigidas hacia el logro de un objetivo (interpretación y comprensión) en las atletas, en aras de formar la conciencia, desarrollar costumbres y hábitos de conducta.

Se evidencio en el entrenamiento las insuficiencias al realizar los ejercicios en los diferentes niveles e inadecuadas posiciones adoptadas durante la ejecución de las coreografías, dificultades en la coordinación por lo que llegan a ser torpes en algunos momentos y mostrar movimientos excesivos que causan en el espectador indicios de burla, risas por lo que se les incentiva a la preparación, constancia, disciplina y mucho apoyo.

Utilizando materiales de apoyo y un folleto asequible en cuanto al lenguaje y comprensión del mensaje que tiene que ser claro, directo, capaz de orientar y dar sentido a la acción o actividad, se les orienta tanto a ellas como a su familia cuando están en presencia de un abusador, acosador u otra persona que intenta maltratarla.

En la realización de esta labor educativa la cual es un proceso complejo, que se realiza desde el presente y aspira al futuro. Requiere paciencia, preparación, superación sistemática profesional, compromiso con la tarea, sensibilidad con esta necesidad educativa especial; para lograr una influencia positiva en las mismas y merecer su respeto. De esa manera es que se puede lograr la unidad entre los conocimientos, los valores y el desarrollo físico de estas.

El éxito de la labor educativa depende de la influencia de muchos factores y condiciones (internas y externas). Estas acciones educativa -terapéuticas se caracterizan por ser dinámicas, activa, flexible, dialéctica y ascendente; además, la motivación por el cumplimiento del objetivo y el ejemplo personal de la entrenadora y profesores son decisivos para el éxito de la misma.

Se tuvo en cuenta las características de estas familias ya que los padres de estas atletas son de aprendizaje lento, con bajo nivel económico, algunos son retrasados, no están activos en la actividad laboral y solo una es graduada de técnico medio y es la que participa en las actividades, por lo que se hace necesario orientar estas acciones de manera individual para que la explicación fluya y sea entendible por estos. Se les orientó de la mejor manera, con apoyo visual, ejemplos sencillos y fáciles de asimilar y comprender, además se les entregó un folleto.

Con estas acciones se trabaja la parte terapéutica en la rehabilitación de las deformidades posturales que presentan para elevar su autoestima, se les corrige la postura y así elevar la cultura postural desde cualquier espacio de la vida cotidiana.

\section{Recuerda}

No hay ningún motivo para maltratar a una adolescente. Tenemos derecho a que nos traten bien.

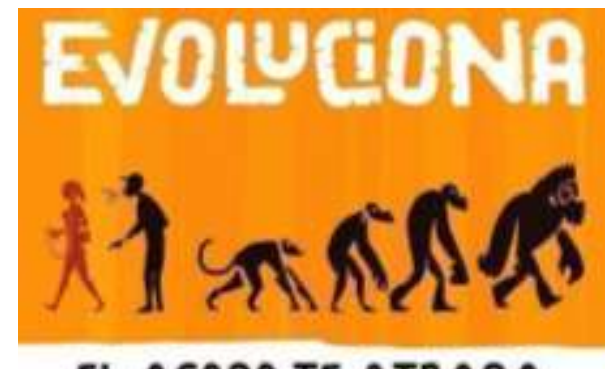

EL ACOSO TE ATRASA

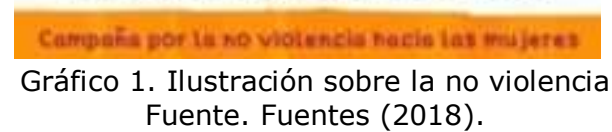

Violencia es hacer daño a una persona o a tus cosas.

Por ejemplo:

Volumen 1 | número 2 | julio-diciembre | 2018 
- Abusar

- Maltratar

- Agredir

- Obligar

- Pegar

- Engañar

\section{Violencia física}

Por ejemplo cuando alguien:

- Te da golpes

- Te pega

- Te da un alón de pelo

- Te tira al suelo

\section{Violencia psicológica}

Otras formas de violencia que no se ven pero duelen mucho.

Por ejemplo cuando alguien:

- Se burla de ti cuando estas practicando la Gimnasia Rítmica.

- Te mira mal haciendo muecas o torciendo los ojos

- No te escucha haciéndose el sordo.

- Te critican todo el rato, que realizaste mal la coreografía se burlan si pierdes en la competencia

- Te insultan con palabras ofensivas

- Te obligan a cortar el pelo.

- No te piden tu opinión para hacer algo que no te gusta

- No quiere que hables ni que estés con otras personas.

- Te obligan a vivir donde no quieres u con otras personas.

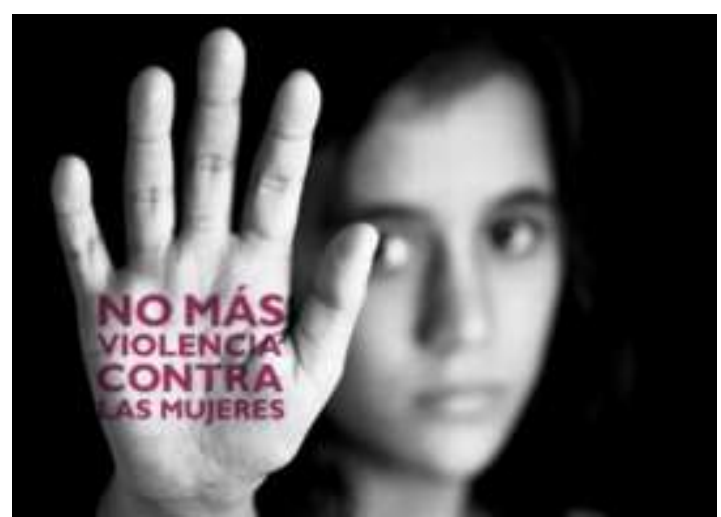

Gráfico 2. Ilustración sobre la no violencia femenina Fuente. Miranda (2018).

También hay formas de violencia sexual.

Por ejemplo cuando otra persona hombre o mujer.

- Toca tu cuerpo o tus partes íntimas sin tu permiso.

- Te obliga a tocar su cuerpo.
- Te pide que realices algún ejercicio de la Gimnasia rítmica como el sapito, sprit

Las personas abusadoras te engañan para conseguir algo,

- dice mentiras

- Te regala cosas para que te calles la boca

- Te toca y tú no quieres.

- No te deja tranquila

Estas personas pueden ser hombres o mujeres que tú conozcas.

Por ejemplo:

- Tu novio.

- Amigos o amigas

- Vecinos o vecinas

- Alguien de la familia (tío, hermano)

\section{Qué no hacer}

Cuando alguien te pida que le bailes, o muestres lo que haces en el deporte dile no.

Te invitan a pasear dile no.

La ropa de tu gimnasia no la uses fuera del entrenamiento.

\section{A quién pedir ayuda?}

A tus padres, entrenadora, maestras, directora de la escuela

\section{RESULTADOS}

Se utilizó la persuasión y el convencimiento, como métodos esenciales siendo los principales recursos el debate, el diálogo y la argumentación. Una vez aplicado las acciones educativa-terapéuticas se realiza un segundo protocolo de observación en el cual se observó que los entrenamientos tienen buena calidad en cuanto a la corrección de la postura y el trabajo educativo. Las atletas presentan buena conducta postural al iniciar los entrenamientos con su debida posición y mantenimiento de la misma. En la ejecución de los ejercicios la entrenadora se ocupa de la correcta posición inicial para la realización de la coreografía, les corrige la postura a las atletas y argumenta sobre los beneficios o perjuicios que trae como consecuencias la mala postura además para la estética y belleza del cuerpo.

Las atletas se encuentran motivadas en todo momento del entrenamiento, la entrenadora las estimula y tienen mejor concentración al realizar los ejercicios y la coreografía. En la competencia se observó la ejecución coreográfica con un mayor nivel atendiendo a las características de su discapacidad. En cuanto al mensaje transmitido en el folleto lo 
entienden $y$ se muestran más seguras $y$ confiadas por el apoyo de la familia, la entrenadora y profesores de la escuela. Además la actitud ante los espectadores es diferente en cuanto mantienen la distancia particularmente hacia los varones.

\section{ANÁLISIS Y DISCUSIÓN}

Con la realización de este trabajo se afirma que el trabajo educativo en la discapacidad intelectual, y de manera particular desde la práctica del deporte Gimnasia Rítmica, es toda actividad física organizada y dirigida a las estudiantes, a partir del desarrollo físico-motor, para mejorar la imagen, lograr belleza en los movimientos y orientación espacial, así como mejorar su desempeño, la expresión de los sentimientos y el lenguaje corporal; para dejar en la conciencia de las mismas una huella sobre lo bueno, malo, bello, feo, correcto e incorrecto de sus acciones o las de los otros en el medio social.

En estudios bibliográficos analizados se aborda el tema de la violencia en la mujer discapacitada en nuestro país, pero son escasos los estudios en la discapacidad intelectual como tal y menos la adolescencia. No se abordan temas sobre el trabajo educativo para prevenir la violencia desde el deporte, además no existe una organización que los represente y atienda como es el caso de la ANSOC, ANCI y ACLIFIM.

En el ámbito internacional existen programas para identificar la violencia en la mujer con discapacidad intelectual y estrategias de apoyo en países como España. Esta investigación continúa en otros espacios tanto en el ámbito escolar como en el deporte se espera que ellas puedan identificar, reconocer signos de acoso sexual, de maltrato físico y psicológico.

Esta labor educativa es un proceso complejo, que se realiza desde el presente y aspira al futuro, requiere paciencia, preparación, superación sistemática profesional, compromiso con la tarea, sensibilidad con esta necesidad educativa especial; para lograr una influencia positiva en las mismas y merezcan respeto.

\section{CONCLUSIONES}

El trabajo educativo para prevenir la violencia en adolescentes atletas con discapacidad intelectual moderada realizado en el proceso de entrenamiento de la Gimnasia rítmica mediante acciones educativa- terapéuticas contribuye a detectar, caracterizar, enfrentar y denunciar actos e indicios que engendran violencia tanto física como psicológica.

Las atletas con la implementación de estas acciones lograron una notable mejoría en su actitud ante el entrenamiento en cuanto a la confianza, belleza en sus movimientos, en la forma de vestir, en la relación con sus compañeros preferentemente varones. El nexo afectivo entrenadora-atleta contribuye a su seguridad, familiaridad, además de expresar sus necesidades, aspiraciones, sueños y se logró mitigar el maltrato y asedio de hombres que la buscaban.

\section{REFERENCIAS BIBLIOGRÁFICAS}

Barrios, L. (2002) III Conferencia Mundial contra o Racismo. Revista Estudios Feministas $(10,1)$ Universidad Federal de Santa Catarina.

Cobas Ruiz, M. (2010) La investigación-acción en la atención de las personas con discapacidad en las repúblicas de Cuba y bolivariana de Venezuela. Tesis (Doctor en Ciencias de la Salud). Editorial Universitaria. La Habana

Cubamotricidad (2018). Persiste la discriminación de la mujer en el deporte. Intervención evento

Colectivo de autores. (2017) Guías de Entrenamiento Gimnasia Rítmica de Olimpiadas Especiales.

Chamero, M. (2016). Discapacidad intelectual | Plena inclusión. Recuperado de www.plenainclusion.org/discapacidadintelectual.

Fuentes, T. (2018). El acoso te atrasa", una campaña cubana por la no violencia contra la mujer. Cuba Debate. Contra el terrorismo mediático. Recuperado de: http://www.cubadebate.cu/noticias/2018 /11/27/el-acoso-te-atrasa-unacampana-cubana-por-la-no-violenciacontra-la-mujer-video-ypdf/\#.X8Ve1GhKgdV

Volumen 1 | número 2 | julio-diciembre | 2018 


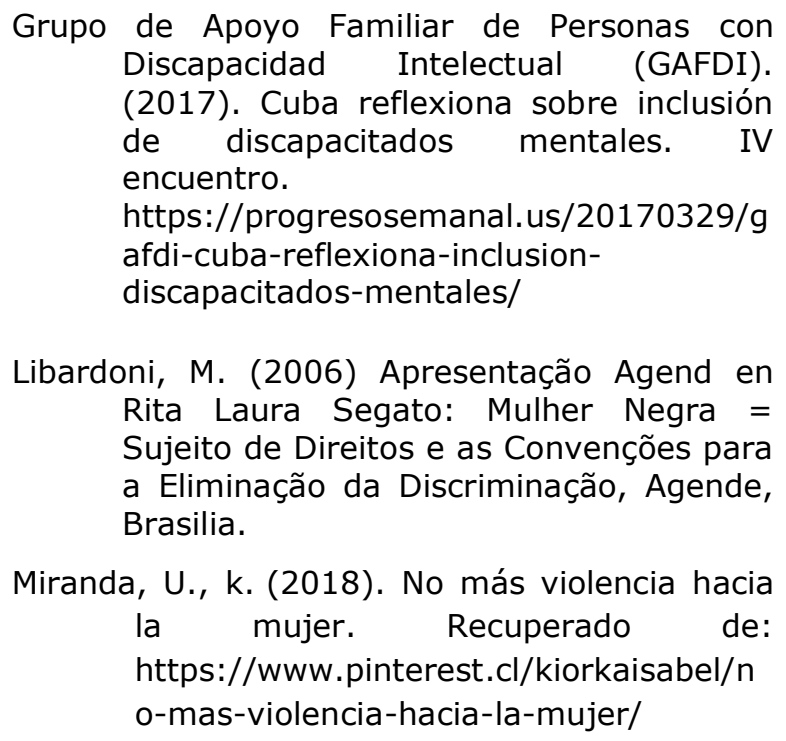

Libardoni, M. (2006) Apresentação Agend en Rita Laura Segato: Mulher Negra = Sujeito de Direitos e as Convenções para a Eliminação da Discriminação, Agende, Brasilia.

Miranda, U., k. (2018). No más violencia hacia la mujer. Recuperado de: https://www.pinterest.cl/kiorkaisabel/n o-mas-violencia-hacia-la-mujer/ 